\title{
AUTOMATED HYDROPONICS SYSTEM USING NFT SYSTEM AND IOT
}

\author{
MALCOLM ANDREW MADEIRA, ADITYA UDAY PAI, ASMITA ASHISH MAHAMBREY,
} ARCHA ABHAY KARANDE, ANISH ASHOK VERLEKAR, VALERIE MENEZES \& BASIL JOSE

Department of Computer Engineering, Agnel Institute of Technology and Design, Assagao-Goa, India \begin{abstract}
In the urban areas there is lack of open green space for farming and even if the land is available it is infertile for plants to grow on them. Problems faced in urban areas farms are due to the toxic elements let in the soil. The sources of toxic metals and effluents in urban soils are mainly from emissions from industries, automobiles, industrial as well as domestic sewage and also. In urban areas people are busy in their work which leads them to buy pesticide and chemically treated food which in injurious to health and they are unable to grow organic vegetable at home due to deficit of space, time and un-fertile soil. In hydroponics we grow the plant without using the soil medium. In this technique we ensure that the roots of the plants absorb the required nutrients from the nutrient solution. The NFT is the most efficient and most popular hydroponics technique type. In this technique we have grown the plants by supplying the nutrient to the root of the plant using water channels made up of pipes until the plants harvested. Our automated system is developed using ESP32S (38 pins) and all the functions of the hydroponics system can be controlled through an app and a website with minimal manual user interference.

The major problem with the manual system is manually checking the contents of nutrients in water, temperature, humidity and the flow of water. The hydroponics monitoring and automation system that is monitored using sensors and microcontrollers. In this system the person sitting at his work place can monitor the hydroponics plant with minimal manual human assistance.
\end{abstract}

KEYWORDS: NFT, Hydroponics \& ESP32S

Received: May 02, 2019; Accepted: May 22, 2019; Published: Jun 01, 2019; Paper Id.: IJCSEITRJUN20197

\section{INTRODUCTION}

Hydroponicsis a technique of growing different varieties of plants or vegetables without using the medium of soil (Soil-less Medium) but using mineral or nutrient solutions mixed with water and on the technique of hydroponic system used, the plant's roots are dipped in water streams or misted with the nutrient mixed water solution in a way such that the plant can absorb the elements it needs for its growth cycle. In this project the aim is automating the process of hydroponics systems using ESP32S Microcontroller along with different sensors and high-power devices. Our system is based on automating the hydroponics system. The hydroponics farming ecosystem consists of four parts, they are as follows:

- The Hydroponics farming technique i.e. NFT

- $\quad$ The detection sensors

- The control system. 
- The lighting system.

- Water cycle system.

- Nutrient dispel system.

- Temperature control system.

- Interfacing of sensors to ESP 32S.

- Interfacing of high-power devices.

- Notification system

- Webpage or a simple user APP

- $\quad$ Taking user intervention.

\section{BACKGROUND STUDY}

\section{The Manual Hydroponics System using NFT}

This NFT method is used for developing green leafy plants with short heights. It's called as Nutrient Film Technique because a thin and constricted flow of water moves over the channel and the nutrient stream forms thin film at the bottom. The channels are kept slopping for the nutrient film to easily flow. Plants are first grown in the nursery and transplanted into the channels. To transplant into the channels net pots are mostly used along with a bit of growing medium to hold the toots in place at the beginning. One can design NFT system in many ways but there are few components that every system should have:

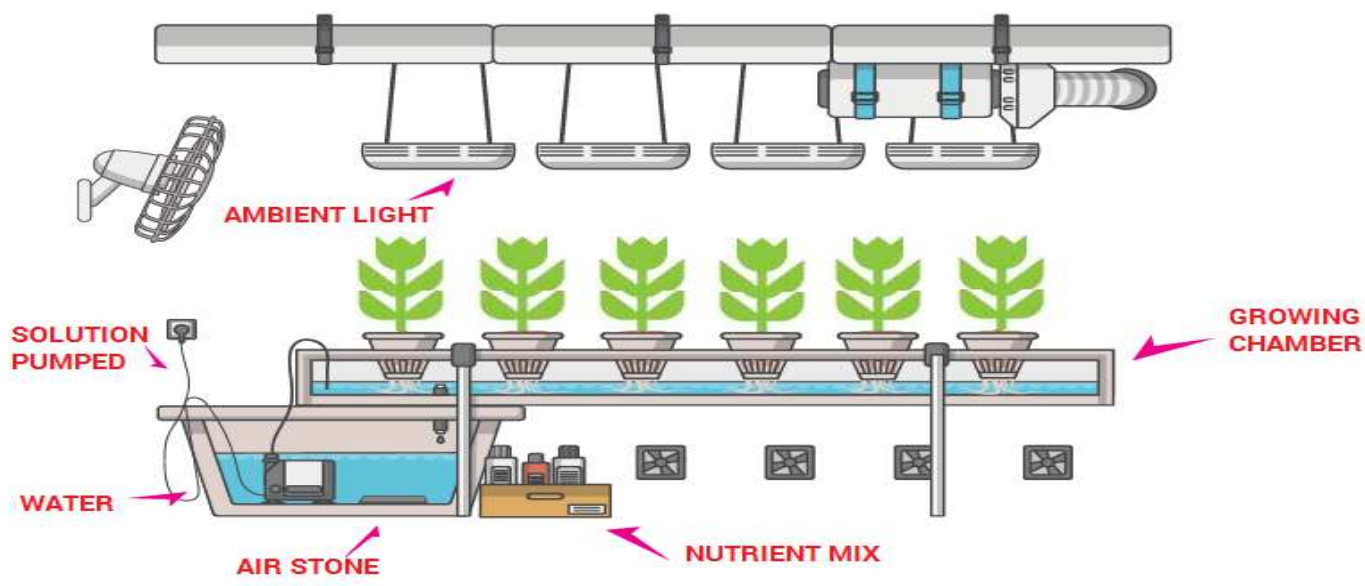

Figure 1: 1Manual Hydroponics System

- Reservoir: Here the water is blended with nutrients in proper proportion before providing to the plants. It is placed beneath the water channels and water is siphoned upwards to the channels.

- Air Pump: It is utilized to aerate the water in other words to oxygenate it in the reservoir with a steady wind flow output.

- Air Stone: It has a miniaturized pore design which stimulates the developing solution as it oxygenates and circulates the nutrients. 
- Water Pump: Water pump is essentially used to transfer water from the source of water to the repository whenever water in the reservoir goes underneath some specific level and also to pump water from the reservoir to the water channel to feed the plants.

- Net Pots: These are utilized to advance bigger and healthier roots. It is easy to maintain cleanliness of the net pots. This container makes it easier to snatch, lift and relocation of the plants.

\section{Nutrient Film Technique}

\section{Working of NFT System}

In consistent solution culture arrangement, the nutrient solution ceaselessly flows through the roots. It is a lot less demanding to computerize than the static arrangement culture because inspecting and changes of $\mathrm{pH}$ and $\mathrm{EC}$ to the temperature and nutrient concentrations can be developed in a huge volume of storage tank that can serve a huge number of plants.

A well-known variety of hydroponics is Nutrient film method (NFT). Where an extremely shallow flow of water containing the nutrient solution required for development of plants is recirculated through the uncovered roots of plants in form of small water channels, a plentiful supply of oxygen is given to the roots of the plants as the roots are suspended above the water stream.

An appropriately structured NFT framework depends on utilizing the correct channel slope, the right stream rate, and the right channel length. The most prominent benefit of this system over other techniques is that the plant roots are presented to satisfactory supply of water, oxygen, and nutrients.

NFT system because of its structure, facilitates a system where all the prerequisites for healthy plant development are met. The outcome of this is that greater production of high-quality plant produce are obtained. A downside of NFT is that it has very few buffering against intrusions in the stream on the account of power failure or blockages in the stream of water channel.

\section{N.F.T. System Flow Rate, and Channel Slope}

The incline of the channel administers how quick the water flows in the nutrient channel. The suggested incline for a N.F.T. framework is typically a 1:33 to 1:43 ratio. That is for every 33 to 43 inches of horizontal length, one inch of drop (incline) is suggested. When planning to design N.F.T. systems, it ought to be structured so that one can slant or incline the slope while the plants are as yet developing. This is due to the root frameworks gets bigger, they may cause it to pool and dam up the water stream. In the situation such that it's movable you can tilt it more to redress if necessary.

\section{Advantages}

- Compared to other techniques, plants grown under NFT technique yield a better growth.

- $\quad$ Space requirement in NFT technique is less.

- Lower water and Nutrient consumption.

- Prevents localized accumulation of salt build up. 


\section{Disadvantages}

- Water pipes at times may get blocked if not properly maintained.

- This technique cannot be used to grow medium to large plants (tap roots).

Table 1: Existing Automated Hydroponics System

\begin{tabular}{|c|l|}
\hline S. No & \multicolumn{1}{|c|}{ Features } \\
\hline 1. & $\begin{array}{l}\text { All parts are automated except nutrient mixing } \\
\text { Rupees:102631(Controller) } \\
\text { Rupees:185288 (Grow Box) }\end{array}$ \\
\hline 2. & Limited types of crops can be grown. \\
\hline 3. & Hardware cost is more for similar setup. \\
\hline 4. & Nutrient solution needs to be pre-prepared. \\
\hline 5. & Power consumption is high. \\
\hline 6. & Maintaining a system is difficult \\
\hline 7. & High Cost of Maintenance. \\
\hline 8. & User interface is not user friendly. \\
\hline
\end{tabular}

\section{PROBLEM DEFINITION}

Our research project is based on Hydroponics System, we are using a common technique, NFT (Nutrient film technique.) The existing system is all manual, that is human being is required to be present at all times. It is because, there is no automation involved in large scale whatsoever. All tasks are carried out manually including checking of $\mathrm{pH}$, electric conductivity also known as (EC), checking for the right water levels in reservoir, turning on of light sources is also done manually also switching up of high voltage devices such as air pumps, water pumps etc. are foreseen by a human. This project is developed to replace human power to detect and recognize the farming domain.

\section{Project Specification}

The modification of the existing manual system by automating it to suit our needs. Automation includes keeping a track of varying $\mathrm{pH}, \mathrm{EC}$ levels, water levels and also turning on and off of the high voltage appliances like air and water pump, exhaust fan etc. by using sensors. Microcontroller ESP32s is used to interface all these sensors. This microcontroller has inbuilt Wi-Fi module which is used to communicate between sensors and web interface using IOT (Internet of things)

These sensors are

- $\mathrm{PH}$ sensor for measuring the $\mathrm{pH}$ of the solution in the reservoir.

- EC sensor is used to check the electrical conductivity of the nutrient solution.

- Water level sensor is used to measure the level of water in the reservoir.

- LDR sensor is used to check if the lights are ON or OFF.

- Water flow sensor is used to check if there is a continuous water flow or no.

- Relay is used as an automatic switch to control high voltage devices like LED lights, water-pump, air pump etc. which are above $5 \mathrm{v}$ since the microcontroller ESP 32 s cannot supply above $5 \mathrm{v}$.

- DHT11 and DS18B20 measures the temperature of its surrounding. 
- C Programming language is used to program the ESP32. All the values measured by the sensors through microcontroller will be displayed on the web interface and an app using which the user can take necessary actions on the hydroponics system.

\section{Block Diagram of Hydroponics System}

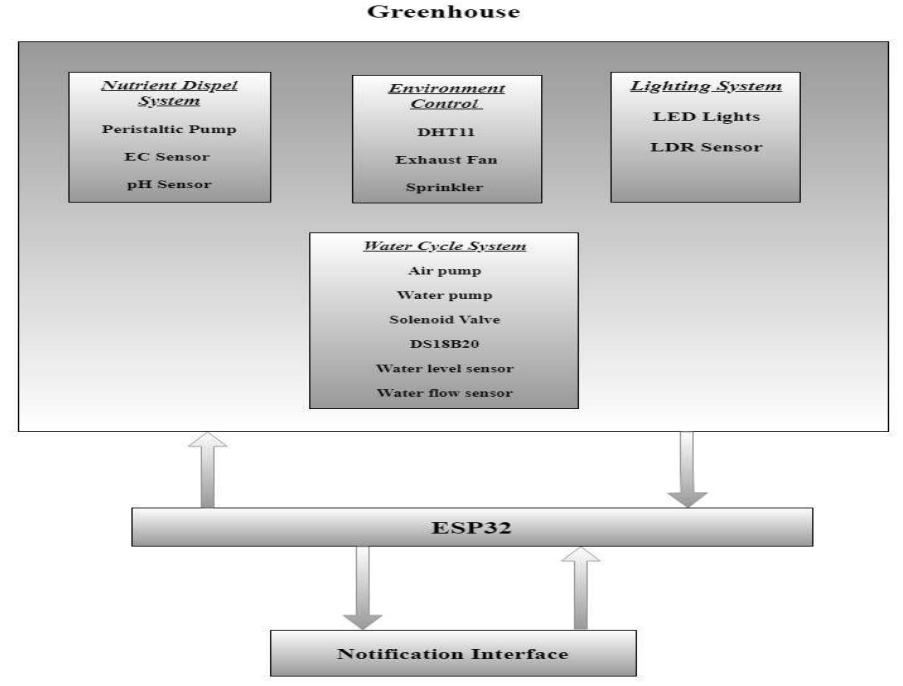

Figure 2: Block Diagram of Hydroponics System

In this block diagram there are four modules which are programmed using Arduino IDE they are Nutrient dispel system, Environment control system, Lighting control system and water cycle control system and all the modules are flashed in ESP32 microcontroller and can be controlled using the web interface.

The system also has programmed notification interface which sends the user messages regarding the parameters of the system and if any condition is violated a user alert is sent to the user via sms.

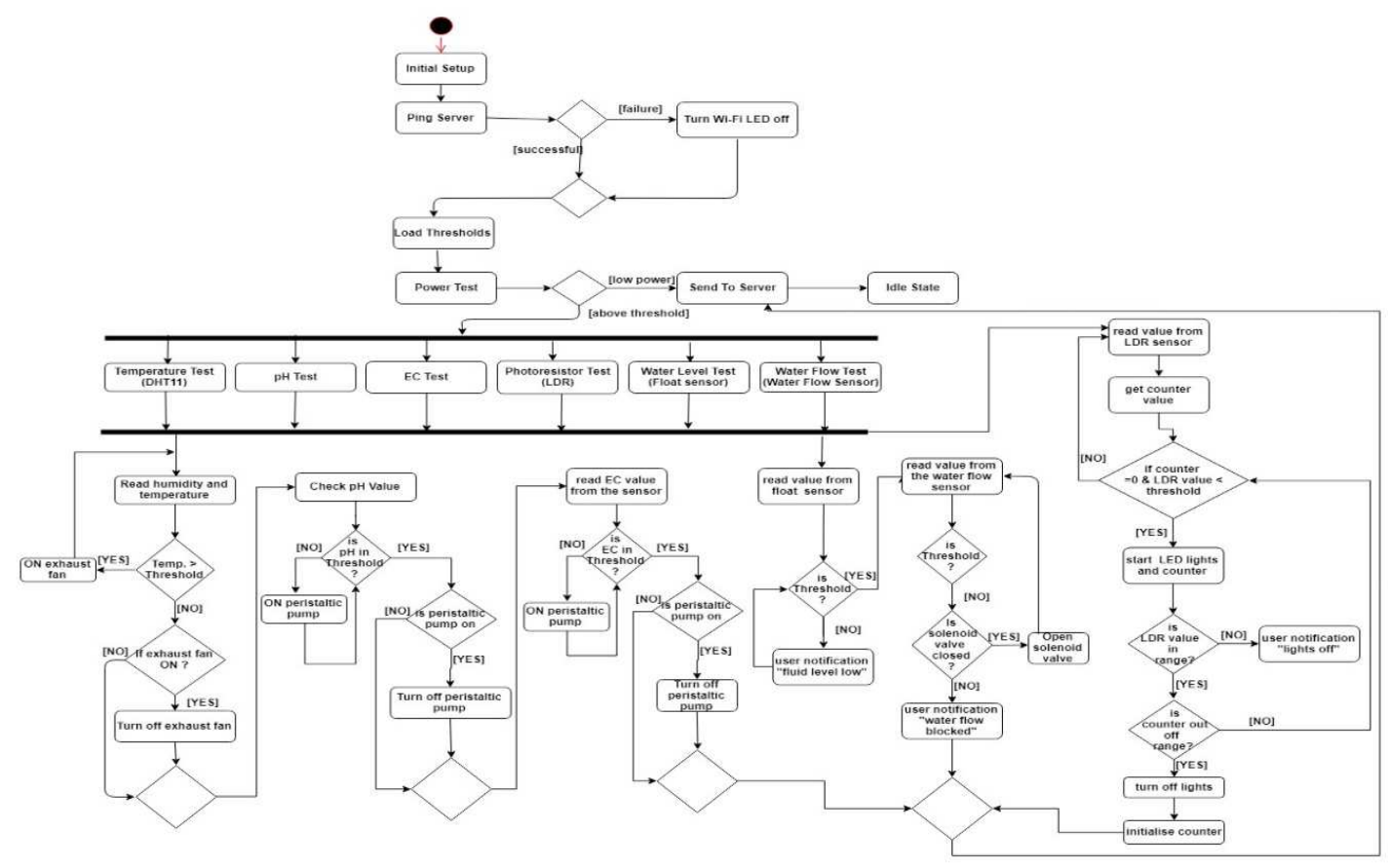

Figure 3: System Activity Diagram 


\section{RESULTS AND PROTOTYPE}

In our automated Hydroponics System using ESP32S all the sensors were interfaced successfully. There were four modules created they are Environment control Module, Nutrient Dispel System, Lighting System and water Cycle system were implemented along with user interface in the form of website and app.

Table 2

\begin{tabular}{|c|l|c|c|}
\hline S. No & \multicolumn{1}{|c|}{ Item Type } & Quantity & Amount \\
\hline 1. & Prototype & 1 & 7000 \\
\hline 2. & NFT Channel & 1 & 650 \\
\hline 3 & Exhaust Fan & 1 & 1600 \\
\hline 4 & Air flow fan & 1 & 450 \\
\hline 5. & Water Pump & 1 & 190 \\
\hline 6. & LED (5-big,2-small) & 7 & 2230 \\
\hline 7. & Switch Board & 1 & 880 \\
\hline 8. & Tray and Solution utensil & 1 & 550 \\
\hline 9. & Air Pump & 1 & 410 \\
\hline 10. & Pipes (5mts) & 1 & 85 \\
\hline 11. & ESP32 & 1 & 1191 \\
\hline \multicolumn{2}{|c|}{ Total } & & $\mathbf{1 5 , 2 3 6}$ \\
\hline
\end{tabular}

\section{Cost of the Project}

Table 3: Cost of the System

\begin{tabular}{|c|l|c|c|}
\hline S. No & \multicolumn{1}{|c|}{ Type (Sensors) } & Quantity & Amount \\
\hline 1 & pH Sensor & 1 & 5617 \\
\hline 2 & EC Sensor & 1 & 5617 \\
\hline 3. & Relay & 1 & 420 \\
\hline 4 & Water flow sensor & 1 & 310 \\
\hline 5 & $\begin{array}{l}\text { DHT11 Temperature \& } \\
\text { Humidity }\end{array}$ & 1 & 188 \\
\hline 6 & $\begin{array}{l}\text { DS18B20 Temperature } \\
\text { Sensor }\end{array}$ & 1 & 525 \\
\hline 7 & LDR Sensor & 1 & 178 \\
\hline 8 & Float Sensor & 3 & 717 \\
\hline \multicolumn{2}{|c|}{ Total } & & $\mathbf{1 3 , 5 7 2}$ \\
\hline \multicolumn{2}{|c|}{} \\
\hline
\end{tabular}

\section{Prototype}

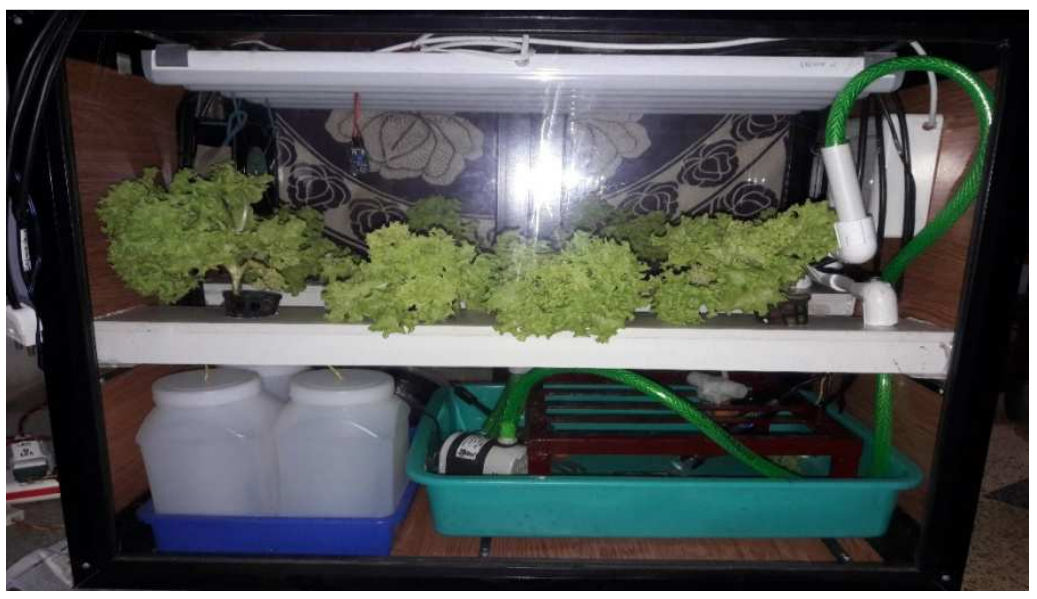

Figure 4: Hydroponics System Prototype with Lettuce Plants Grown 

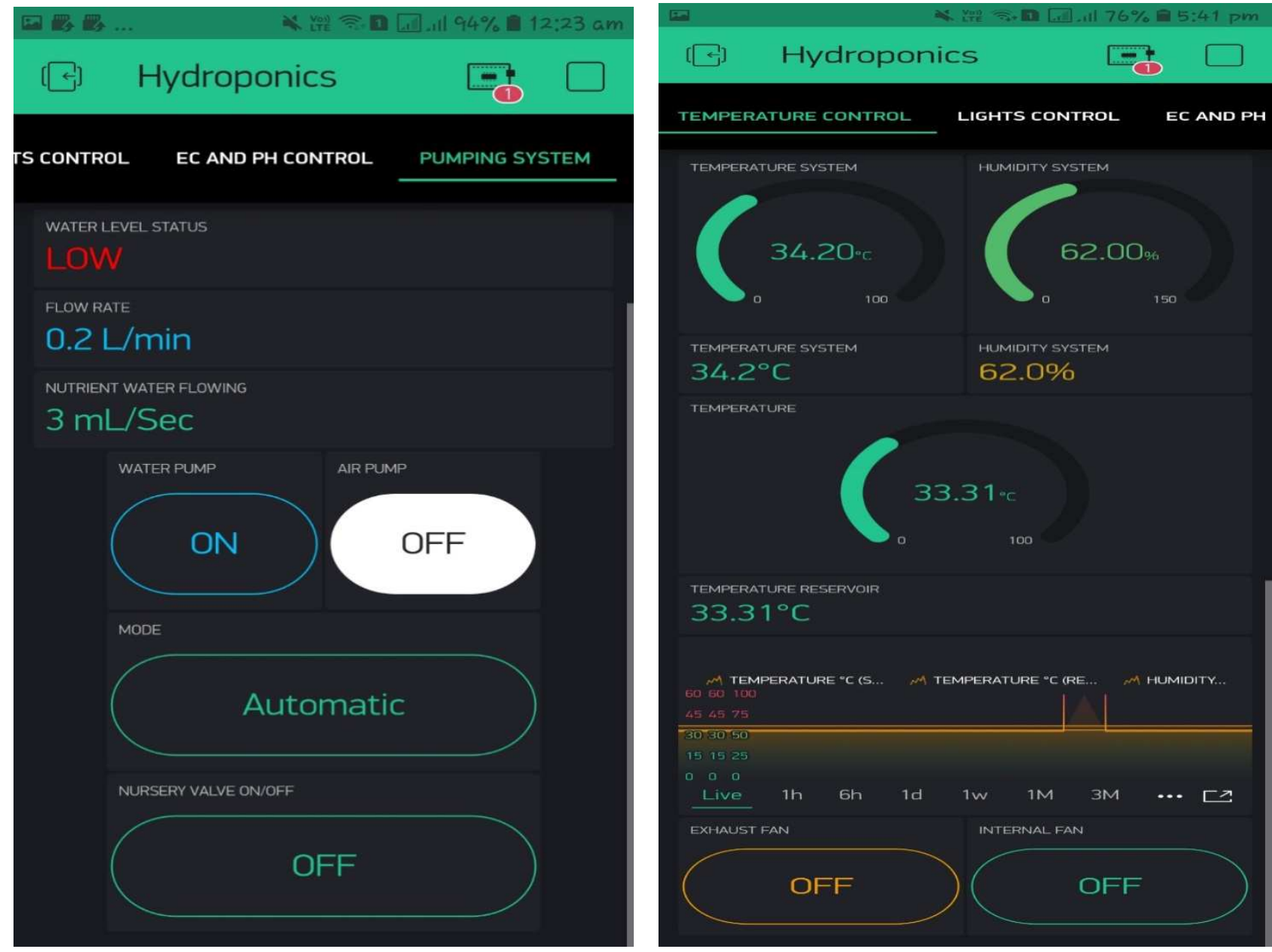

User Interface
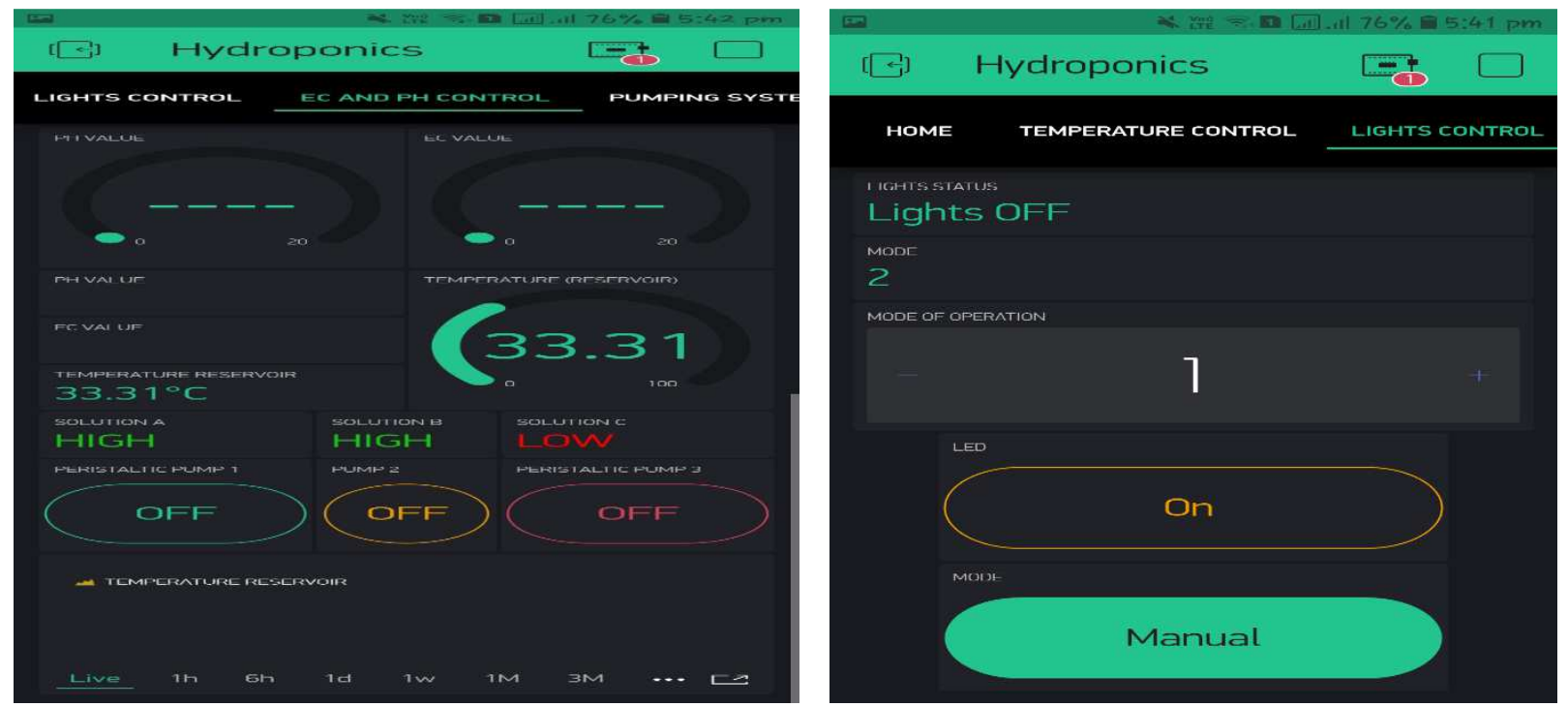

Figure 5: User App

\section{DISCUSSIONS}

In this automated Hydroponics system, the user has to log in in "Blynk" platform to open the app with the QR code of the project once this is successfully performed the user can view the Hydroponics system parameters. In the first 
page the user can view the Temperature control system and can control the systems temperature and humidity by switching on/off the Exhaust/internal fan using this app. The DHT11 sensor is interfaced on GPIO pin 13 of the ESP32 and the DS18B20 is interfaced on the GPIO pin 26 of the ESP32 and can also view the data in the form of graph for comparison. The next page is the pumping system, here the user can check if water is flowing in system via the water flow sensor data given as flow rate and nutrient water flowing parameters and also the water level of the reservoir, here the user can select the manual or automated mode for controlling the water pump and air pump. The water pump and the air pump are interfaced to GPIO pin 19 and 18 respectively and the water flow sensor to the GPIO pin 15 and water level sensor to GPIO pin 14 of ESP32. In the EC and pH control system the user can check the EC and pH of the water solution in the reservoir and also the status of the water and nutrient levels via the water level sensor and the user can adjust the $\mathrm{pH}$ and EC by switching on/off the peristaltic pumps. The EC and pH sensor are interfaced to GPIO pin 36 and 29 respectively and the water level sensor is interfaced to GPIO pin 14,25 and 33 and the three pumps to GPIO pins 16,17 and 32 respectively. In the lights control system the user can switch between manual or automatic mode and can select the 3 modes from 0 to 1 and can also see the status of LED lights as on/off via the LDR sensor, LDR sensor is interfaced on GPIO pin 39 and the LED lights are interfaced on GPIO pin 23.

In this automated system the user outside its local area network using internet can access the system and can control all the parameters of the automated System remotely and also from outside of India.

\section{CONCLUSIONS}

This research was focussed on interfacing and integrating all the functions of the Hydroponics system together using the ESP32 microcontroller which is easily available at an affordable price. The hydroponics system uses NFT (Nutrient film technique to grow the plants and all the functions of the system can be controlled remotely using wi-fi enabled ESP32. All the sensors were interfaced successfully and the data collected from the sensors can be viewed on the user app and based on the data displayed the user can take appropriate actions without manually being present at the prototype. The user app was developed using Blynk IOT platform and the ESP32 microcontroller was programmed using Arduino IDE.

The cost of the project is including the cost of the prototype is rupees 28,808 and is the cheapest in the market as it has only one microcontroller chip and all the sensors are integrated and can be controlled via an app which has simple user interface.

This project can also be scaled for large farm lands without any changes in hardware and software and the setup of the project is simple.

\section{REFERENCES}

1. (2018, December 10). Retrieved from forum.arduino.cc: https://forum.arduino.cc/index.php? topic $=437519.0$

2. Amazon. (2018, December 10). Retrieved from Component7-LDR-Dependent-Register-Resistor/dp/B01BADCLH0: https://www.amazon.in/Component7-LDR-Dependent-Register-Resistor/dp/BO1BADCLHO

3. Ectrical_conductivity_meter. (2018, December 12$). \quad$ Retrieved from en.wikipedia.org: https://en.wikipedia.org/wiki/Electrical_conductivity_meter

4. Gaurav, N., Singh, A., Srivastava, A., Singh, A., \& Gariya, H. S. in Vitro Propagation and Production of Qualitative Traits of Cultivated Variety of Withania Somnifera L. From Callus of Embryonic Cotyledon Explants in B5 Medium. 
5. https://en.wikipedia.org/wiki/ESP32. (2018, December 10). Retrieved from https://en.wikipedia.org/wiki/ESP32: https://en.wikipedia.org/wiki/ESP32

6. https://wiki.metropolia.fi/display/sensor.

December

10).

Retrieved

from wiki.metropolia.fi/display/sensor/Introduction+to+pH+sensors: https://wiki.metropolia.fi/display/sensor/Introduction+to+pH+sensors

7. Slideshare. (2018, December 10). Retrieved from https://www.slideshare.net/ajaygurumadu/laser-security-system-72827462

8. What-is-dark-resistance. (2018, December 19). Retrieved from https://www.quora.com/What-is-dark-resistance

9. www.china-electricvalves.com/news/news17.html. (2018, December 10). Retrieved from www.china-electricvalves.com: http://www.china-electricvalves.com/news/news17.html

10. www.revolvy.com/topic/Solenoid valve. (2018, December 10). Retrieved from www.revolvy.com/topic/Solenoid valve: https://www.revolvy.com/topic/Solenoid 
